\title{
Meshless Local Petrov-Galerkin Method for Scattering from 2-D Rectangular Cavities in a Ground Plane
}

\author{
Meiling Zhao ${ }^{1,}$,, $\mathrm{Li} \mathrm{Li}^{2}$ \\ ${ }^{1}$ School of Mathematics and Physics, North China Electric Power University, Baoding, China \\ ${ }^{2}$ School of Control and Computer Engineering, North China Electric Power University, Baoding, China
}

Email address:

mzhaomath@163.com (Meiling Zhao), haolily12@163.com(Li Li)

To cite this article:

Meiling Zhao, Li Li. Meshless Local Petrov-Galerkin Method for Scattering from 2-D Rectangular Cavities in a Ground Plane. Applied and Computational Mathematics. Vol. 4, No. 3, 2015, pp. 145-151. doi: 10.11648/j.acm.20150403.17

\begin{abstract}
In this paper, we develop the meshless local Petrov-Galerkin formulation of the scattering from rectangular cavities embedded in a ground plane. The electromagnetic scattering by the cavity is governed by the Helmholtz equation along with Sommerfeld's radiation conditions imposed at infinity. The MLPG method is a truly meshless method wherein no elements or background cells are needed, in either the interpolation or integration. Based on local weak form and the moving least square (MLS) approximation, this truly meshless method is applied to solve the scattering problem. The results of numerical experiments have shown the efficiency and accuracy of the proposed method.
\end{abstract}

Keywords: Meshless Local Petrov-Galerkin Method, Electromagnetic Scattering, 2-D Rectangular Cavities, Moving Least Square Approximation

\section{Introduction}

The electromagnetic scattering by open cavities has attracted much attention by both engineering and mathematical community due to its important industrial and military applications. Examples of cavities include jet engine inlet ducts and exhaust nozzles, cavity-backed antennas, and cracks and gaps in the metallic skin of the aircraft. Because a cavity residing in a scatter can significantly contribute to the overall Radar Cross Section (RCS) of the target, the accurate prediction of the RCS of the cavity is necessary. There is a large literature available on computation electromagnetic scattering of open cavities, see for examples [1-4], and references cited therein.

In this paper, we focus on the RCS of open cavity as shown in Fig. 1. The ground plane and the wall of the open cavity are assumed as perfect electric conductors (PEC), and the interior of the open cavity is filled with non-magnetic materials which may be inhomogeneous. The half space above the ground plane is filled with a homogenous and isotropic medium with its permittivity $\varepsilon_{0}$ and permeability $\mu_{0}$. In this setting, the electromagnetic scattering by the cavity is governed by the Helmholtz equation along with Sommerfeld's radiation conditions imposed at infinity. Classical transparent boundary condition is introduced at the aperture, and the cavity problem defined in an infinite domain is reduced to a Helmholtz equation with simple boundary conditions on the wall of the cavity and a nonlocal boundary condition on the aperture.

The development of new numerical methods for the approximate solutions of Helmholtz equations is an interesting research area of many engineers and mathematicians. Meshless methods, as alternative numerical approaches to the classical numerical methods such as the finite element and boundary element method, have attracted much attention in recent years, because of their flexibility and simplicity. Unlike the conventional numerical methods, meshless methods require neither domain nor boundary discretization and consequently no information on the connectivity between nodal points and elements is needed. In the two last decades, many types of meshless methods have been proposed. Among these meshless methods, the meshless local Petrov-Galerkin method (MLPG) proposed by Atluri and his team in [5-6] is a truly meshless method since it does not need a background mesh for numerical integration. Based on the local weak form of governing equations over small subdomains specified for each nodal point, all integrals can be easily evaluated over the regularly shaped, overlapping subdomains of arbitrary shape and their respective boundaries. Therefore no elements or background cells are necessary either for interpolation or integration. In this paper 
we apply the MLPG method to solve the scattering of electromagnetic plane waves by a two-dimensional (2-D) rectangular cavity filled with the homogeneous medium.

The rest of the paper is organized as follows. In the next section, the governing equation of scattering model from open cavity is described and further is reduced to a bounded domain problem. In the third section, the MLPG method is applied to solve the scattering problem. Numerical experiments are presented to illustrate the competitive behavior of the method in section 4 . The paper ends with some conclusions in the last section.

\section{The Electromagnetic Scattering Model by the Rectangular Cavity}

In this paper, we consider the electromagnetic scattering of an incident plane wave by a two-dimensional open rectangular cavity embedded in an infinite ground plane as shown in Fig.1 Throughout the paper, the medium and material are invariant in the $z$-direction. As shown in Fig. 1, an open cavity $\Omega \in R^{2}$, enclosed by the aperture $\Gamma$ and the wall $\partial \Omega \backslash \Gamma$, is embedded in the perfectly electric conducting ground plane $\Gamma^{C}$. The medium is assumed to be non-magnetic and has a constant magnetic permeability, which equals to the magnetic permeability of vacuum. The medium in the half space above the ground plane is assumed to be homogeneous with positive electric permittivity $\varepsilon_{0}$. The interior of the cavity is filled with homogeneous medium, which have relative electric permittivity $\varepsilon_{r}(x, y)$.

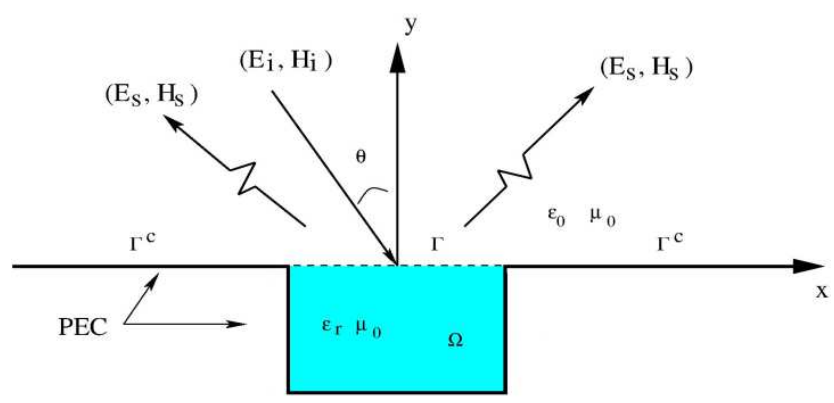

Figure 1. The model geometry of rectangular cavity scattering.

In TM case, the magnetic field is transverse to the invariant direction. The incident and the total electric fields are parallel to the $z$-axis. Assume the incident and the total fields are $E_{i}\left(0,0, u^{i}\right)$ and $E_{t o t}=(0,0, u)$ respectively. By the perfectly electrical conduct condition, the total field $u$ vanishes on the wall and $\Gamma^{C}$. The Maxwell equations are reduced to the two-dimensional Helmholtz equation

$$
\begin{array}{ll}
\Delta u+k^{2} u=f(x, y) & \text { in } \Omega \cup R_{2}^{+} \\
u=0 & \text { on }(\partial \Omega \backslash \Gamma) \cup \Gamma^{C} \\
\mathrm{u}, \frac{\partial u}{\partial n} & \text { are continuous on } \Gamma,
\end{array}
$$

where $k^{2}=\omega^{2} \varepsilon \mu_{0}, \omega$ is the angular frequency and $k$ is called wave number.

Let an incoming plane wave $u^{i}=e^{\mathrm{i} k_{0}(x \sin \theta-y \cos \theta)}$ be incident on the cavity from above space, where $\theta \in(-\pi / 2, \pi / 2)$ is the angle incidence with respect to the positive $y$-axis, and $k_{0}=\omega \sqrt{\varepsilon_{0} \mu_{0}}$ is the wave number of the free space. Assume $u^{s}$ be the tangential component of the scattered field $E_{s}=\left(0,0, u^{s}\right) \cdot u^{s}=u-u^{i}+e^{\mathrm{i} k_{0}(x \sin \theta+y \cos \theta)}$, which satisfies

$$
\Delta u+k_{0}^{2} u=0 \text { in } R_{2}^{+}
$$

In addition, the scattered field satisfies the radiation condition

$$
\lim _{r \rightarrow \infty} \sqrt{r}\left(\frac{\partial u^{s}}{\partial n}-\mathrm{i} k_{0} u^{s}\right)=0
$$

Using the upper half-plane Green's function for the Helmholtz equation, we can get the so-called transparent boundary operator

$$
I(u)(x):=\frac{\mathrm{i} k_{0}}{2} \int_{\Gamma} \frac{1}{\left|x-x^{\prime}\right|} H_{1}^{(1)}\left(k_{0}\left|x-x^{\prime}\right|\right) u\left(x^{\prime}, 0\right) d x^{\prime} .
$$

The scattering problem is reduced to the following bounded domain problem.

$$
\begin{array}{ll}
\Delta u+k_{0}^{2} \varepsilon_{r} u=f(x, y) & \text { in } \Omega \\
u=0 & \text { on } \partial \Omega \backslash \Gamma \\
\frac{\partial u}{\partial n}=I(u)+g(x) & \text { on } \Gamma,
\end{array}
$$

where $g(x)=-2 \mathrm{i} \beta e^{\mathrm{i} \alpha x}$.

In the TE case, the formulation process can be similarly deduced. The total field satisfies

$$
\begin{array}{ll}
\nabla \cdot\left(\frac{1}{\varepsilon_{r}} \nabla u\right)+k_{0}^{2} u=f(x, y) & \text { in } \Omega \\
\frac{\partial u}{\partial n}=0 & \text { on } \partial \Omega \backslash \Gamma \\
u=\tilde{I}(u)+\tilde{g}(x) & \text { on } \Gamma,
\end{array}
$$

where $I(u)(x):=-\left.\frac{1}{2} \int_{\Gamma} \frac{1}{\varepsilon_{r}} H_{0}^{(1)}\left(k_{0}\left|x-x^{\prime}\right|\right) \frac{\partial u\left(x^{\prime}, y^{\prime}\right)}{\partial y^{\prime}}\right|_{y^{\prime}=0^{-}} d x^{\prime}$, $\tilde{g}(x)=2 e^{\mathrm{i} \alpha x}$.

\section{The MLPG Method for the Cavity Scattering Problem}

In this section, we apply the MLPG method for the electromagnetic scattering problem by open rectangular cavities in the case of TM polarization. The algorithm in TE 
case can be similarly formulated.

\subsection{Moving Least Square (MLS) Approximation}

In order to approximate the trial functions over the solution domain, a meshless interpolation scheme is required. The moving least square (MLS) is widely used to interpolate random data with appropriate accuracy in many types of meshless methods for constructing meshless shape functions. The property of MLS has been discussed in many literatures.

In a 2-D domain $\Omega$, we consider a function $u(\mathrm{x})$ with a set of nodes $\left\{\mathrm{x}_{i}=\left(x_{i}, y_{i}\right)\right\}, i=1,2, \ldots, n$, and the parameter associated with the approximation at node $i$ is denoted by $u_{i}$. The MLS approximation $u^{h}(\mathrm{x})$ of $u(\mathrm{x})$ can be defined by

$$
u^{h}(\mathrm{x})=\sum_{j=1}^{m} p_{j}(\mathrm{x}) a_{j}(\mathrm{x})=\mathrm{p}^{T}(\mathrm{x}) \mathrm{a}(\mathrm{x})
$$

where $\mathrm{p}(\mathrm{x})=\left(p_{1}(\mathrm{x}), p_{2}(\mathrm{x}), \ldots, p_{m}(\mathrm{x})\right)$ is usually chosen as complete monomial basis of order $m$. For example,

$$
\mathrm{p}^{T}(\mathrm{x})=\mathrm{p}^{T}(x, y)=\{1, x, y\}, \quad(\text { linear basis, } m=3)
$$

$\mathrm{p}^{T}(\mathrm{x})=\mathrm{p}^{T}(x, y)=\left\{1, x, y, x y, x^{2}, y^{2}\right\}, \quad($ quadratic basis, $m=6)$ in a 2-D model. The coefficient functions in (7) $\mathrm{a}(\mathrm{x})=\left(a_{1}(\mathrm{x}), a_{2}(\mathrm{x}), \ldots, a_{m}(\mathrm{x})\right)$ are determined by minimizing a weighted discrete $L_{2}$ norm, which is defined as follows,

$$
\begin{aligned}
J(\mathrm{x}) & =\sum_{i=1}^{n} w\left(\mathrm{x}-\mathrm{x}_{i}\right)\left(u^{h}\left(\mathrm{x}_{i}\right)-\hat{u}_{i}\right)^{2} \\
& =\sum_{i=1}^{n} w\left(\mathrm{x}-\mathrm{x}_{i}\right)\left(\mathrm{p}^{T}\left(\mathrm{x}_{i}\right) \mathrm{a}(\mathrm{x})-\hat{u}_{i}\right)^{2} \\
& =(\mathrm{Pa}(\mathrm{x})-\hat{\mathrm{u}})^{T} \mathrm{~W}(\mathrm{~Pa}(\mathrm{x})-\hat{\mathrm{u}}) .
\end{aligned}
$$

Here $w\left(\mathbf{x}-\mathbf{x}_{i}\right)$ is the weight function associated with node $i$, and it usually has a local compact support with $w\left(\mathrm{x}-\mathrm{x}_{i}\right) \neq 0$, and $\mathrm{W}=\operatorname{diag}\left(w\left(\mathrm{x}-\mathrm{x}_{1}\right), w\left(\mathrm{x}-\mathrm{x}_{2}\right), \ldots, w\left(\mathrm{x}-\mathrm{x}_{n}\right)\right)$, $\mathrm{P}=\left(\mathrm{p}\left(\mathrm{x}_{1}\right), \mathrm{p}\left(\mathrm{x}_{2}\right), \ldots, \mathrm{p}\left(\mathrm{x}_{n}\right)\right)^{T}$ and $\hat{\mathrm{u}}=\left(\hat{u}_{1}, \hat{u}_{2}, \ldots, \hat{u}_{n}\right)^{T}, \quad \hat{u}_{i} \quad$ is the fictitious nodal value associated with $\mathrm{x}_{i}$. Note the stationarity of $J$ with respect to a(x) leads to the following linear relation between $\mathrm{a}(\mathrm{x})$ and $\hat{\mathrm{u}}$ :

$$
\frac{\partial J}{\partial \mathrm{a}}=\mathrm{A}(\mathrm{x}) \mathrm{a}(\mathrm{x})-\mathrm{B}(\mathrm{x}) \hat{\mathrm{u}}=0,
$$

where the matrices $\mathrm{A}(\mathrm{x})$ and $\mathrm{B}(\mathrm{x})$ are defined by $\mathrm{A}(\mathrm{x})=\mathrm{P}^{T} \mathrm{~W} \mathrm{P}, \mathrm{B}(\mathrm{x})=\mathrm{P}^{T} \mathrm{~W}$. Thus $\mathrm{a}(\mathrm{x})$ can be solved, and we substitute it into (7) and obtain

$$
u^{h}(\mathrm{x})=\sum_{i=1}^{n} \Phi_{i}(\mathrm{x})=\Phi(\mathrm{x}) \hat{\mathrm{u}},
$$

where

$$
\Phi(\mathrm{x})=\mathrm{p}^{T}(\mathrm{x}) \mathrm{A}^{-1}(\mathrm{x}) \mathrm{B}(\mathrm{x}) .
$$

In Eq. (10), $\Phi_{i}(\mathrm{x})$ is usually called the nodal shape function of the MLS approximation corresponding to the nodal point $\mathrm{x}_{i}$. It can be easily seen that $\Phi_{i}(\mathrm{x})$ equals zero when $\mathrm{x}$ is not in the support of the nodal point $\mathrm{x}_{i}$, which preserves the local character of the moving least squares approximation. Furthermore, $\Phi(\mathrm{x})$ is well defined only if the matrix $\mathrm{A}$ is not singular, which means $\mathrm{P}$ must consist of linearly independent row vectors. In addition, a fact is that the smoothness of the shape functions is influenced by the weight functions and basis functions. Let $C^{k}(\Omega)$ be the space of $k$-th continuously differentiable functions. If $w\left(\mathrm{x}-\mathrm{x}_{i}\right) \in C^{k}(\Omega)$, $i=1,2, \ldots, n \quad$ and $\quad p_{j}(\mathrm{x}) \in C^{l}(\Omega), \quad j=1,2, \ldots, m$, then $\Phi_{i}(\mathrm{x}) \in C^{r}(\Omega), r=\min \{k, l\}$. Many kinds of basis functions and the weight functions can be chosen for implementing the MLS approximation in MLPG method, see [6]. In this paper, a spline weight function with compact supports is taken as follows,

$$
w\left(\mathrm{x}-\mathrm{x}_{i}\right)= \begin{cases}1-6\left(\frac{d_{i}}{r_{i}}\right)^{2}+8\left(\frac{d_{i}}{r_{i}}\right)^{3}-3\left(\frac{d_{i}}{r_{i}}\right)^{4}, & 0 \leq d_{i} \leq r_{i}, \\ 0, & d_{i} \geq r_{i},\end{cases}
$$

where $d_{i}=\left|\mathrm{x}-\mathrm{x}_{i}\right|$ is the distance from node $\mathrm{x}_{i}$ to the point $\mathrm{x}$, and $r_{i}$ is the size of the compact support for the weight function $w\left(\mathrm{x}-\mathrm{x}_{i}\right)$ associated with the node $i$. Here, it is obvious that the spline weight function is $C^{1}(\Omega)$ continuous over the entire domain. In order to ensure the regularity of $\mathrm{A}$, $r_{i}$ should be chosen as large enough parameter so as that sufficient number of nodes is covered in the domain of definition of every sample point. However, on the other side, $r_{i}$ should also be not too large so as to maintain the local character of the MLS approximation.

The partial derivatives of the shape functions $\Phi_{i}(\mathrm{x})$ is given by the following

$$
\Phi_{i, s}(\mathrm{x})=\sum_{j=1}^{m}\left(p_{j, s} \mathrm{~A}^{-1} \mathrm{~B}+p_{j}\left(\mathrm{~A}^{-1} \mathrm{~B}_{, s}+\mathrm{A}_{, s}^{-1} \mathrm{~B}\right)_{j i}\right)
$$

where $\mathrm{A}_{s}^{-1}=\left(\mathrm{A}^{-1}\right)_{s}$ represents the derivative of the inverse of $\mathrm{A}$ with respect to $x_{s}$, and it is given by $\mathrm{A}_{s s}^{-1}=-\mathrm{A} \mathrm{A}_{s} \mathrm{~A}^{-1}$, where the index ( $)_{s}$ indicates a spatial derivative.

From the above discussion it can be seen that the MLS shape functions do not possess Kronecker delta property. This will cause the difficulty to impose the essential boundary conditions. Many techniques have been proposed to deal with the difficulty. Lagrange multipliers and penalty methods are two kinds of widely used methods for imposition of essential boundary conditions. However, the use of the Lagrange multipliers will increase the size of linear system and, more 
seriously, the choice of interpolation for the system of multipliers can lead to a singularity in the system, which is hard to predict a priori, see [7]. Though the penalty method requires only a minor modification of the weak form with the introduction of a scalar parameter that controls the imposition of essential boundary conditions, a suitable value for the penalty parameter is not easy to choose in advance. The method presented in [8], by coupling the meshless method and the conventional finite element along the essential boundary, avoid the above drawbacks, and it will be used in this paper.

\subsection{Local Petrov-Galerkin Integral Equation}

Instead of using the global weak form, the MLPG method constructs the weak form over local cell $\Omega_{s}$, which is a small region taken for each node in the global domain $\Omega$. The local cells overlap each other and cover the whole global domain $\Omega$. The local cells could be of any geometric shape and size. In this paper, for simplicity, we take them as circular shapes. The local weak form of the government equation in TM case can be written as follows,

$$
\int_{\Omega_{s}}\left(\Delta u+k^{2} u-f\right) v d \Omega=0
$$

where $\Omega_{s}$ is a local sub-domain associated with any node, and $v$ is a test function. Using the divergence theorem, the above formula yields the following equation

$$
\begin{aligned}
& -\int_{\Omega_{s}} \nabla u \nabla v d \Omega+\int_{\Omega_{s}} k^{2} u v d \Omega+\int_{\Gamma_{s q}} I(u) v d \mathrm{~s}+\int_{\Gamma_{s u}} \frac{\partial u}{\partial n} v d \mathrm{~s} \\
= & \int_{\Omega_{s}} f v d \Omega-\int_{\Gamma_{s q}} g v d \mathrm{~s},
\end{aligned}
$$

Where $\Gamma_{s u}$ is the intersection of $\partial \Omega \backslash \Gamma$ and the boundary $\partial \Omega_{s}$, which is the boundary of $\Omega_{s}$, and $\Gamma_{s q}$ is the intersection of the aperture $\Gamma$ and the boundary $\partial \Omega_{s}$. Different local test functions can be used in the weak form Eq. (15), which leads to different ways to construct the global stiffness matrix, see [6]. Here in Eq. (15), the term $\int_{\Gamma_{s q}} I(u) v d \mathrm{~s}$ needs to be specially considered. Note the definition of $I(u)$, and we can rewrite Eq. (4) as

$$
\begin{aligned}
I(u)(x)= & -\frac{k_{0}}{2} \int_{\Gamma} \frac{u\left(x^{\prime}, 0\right)}{\left|x-x^{\prime}\right|} Y_{1}\left(k_{0}\left|x-x^{\prime}\right|\right) d x^{\prime} \\
& +\frac{\mathrm{i} k_{0}}{2} \int_{\Gamma} \frac{u\left(x^{\prime}, 0\right)}{\left|x-x^{\prime}\right|} J_{1}\left(k_{0}\left|x-x^{\prime}\right|\right) d x^{\prime},
\end{aligned}
$$

where $J_{1}(z)$ and $Y_{1}(z)$ are the first and second Bessel functions separately, and the Hankel function $H_{1}^{(1)}(z)=J_{1}(z)+\mathrm{i} Y_{1}(z)$. Note $Y_{1}(z)$ is hypersingular, so the first part in the above equation denotes a Hadamard principle value integral. By using Toeplitz type approximation in [9] and the classical Toeplitz rule, $I(u)$ in the nonlocal boundary condition (5) can be approximated as

$$
I(u)\left(x_{i+n-M}, 0\right) \approx \sum_{l=1}^{M} G_{i l} u\left(x_{l+n-M}, 0\right) .
$$

Here we assume that $M$ denotes the number of discretization nodes in the horizontal direction of the cavity, and $\mathrm{N}$ is the number of nodes in the longitudinal direction of the cavity, thus $n=N \times M$. Here G is a complex matrix, and it is defined as

$$
G_{i l}=G_{i l}^{\mathrm{Re}}+G_{i l}^{\mathrm{Im}},
$$

Where $G_{i l}^{\mathrm{Re}}$ is the real part, and $G_{i l}^{\mathrm{Im}}$ is the imaginary part,

$$
\begin{gathered}
G_{i l}^{\mathrm{Re}}=-t_{i l} \frac{k_{0}\left|x_{i+n-M}-x_{l+n-M}\right|}{2} Y_{1}\left(k_{0}\left|x_{i+n-M}-x_{l+n-M}\right|\right), \\
G_{i l}^{\mathrm{Im}}=\frac{k_{0} h}{2\left|x_{i+n-M}-x_{l+n-M}\right|} J_{1}\left(k_{0}\left|x_{i+n-M}-x_{l+n-M}\right|\right), \\
t_{i l}= \begin{cases}(1-\ln 2) / h, & |i-l|=1, \\
-1 / h, & i=l, \\
\left(\ln \left((i-l)^{2}\right) /\left((i-l)^{2}-1\right)\right) / h, & \text { otherwise. }\end{cases}
\end{gathered}
$$

Applying the MLS approximation for the unknown functions, and choosing $\Phi(\mathrm{x})$ as the test function $v(\mathrm{x})$, we can transform the local integral (15) to a system of algebraic equations with unknown quantities at nodes used for spatial approximation as follows,

$$
\mathcal{K} \hat{\mathrm{u}}=\mathcal{F}
$$

where

$$
\begin{aligned}
\mathcal{K}_{i j}= & -\int_{\Omega_{s}}\left(\left(\frac{\partial \phi_{i}}{\partial x} \frac{\partial \phi_{j}}{\partial x}+\frac{\partial \phi_{i}}{\partial y} \frac{\partial \phi_{j}}{\partial y}\right)+k^{2} \phi_{i} \phi_{j}\right) d \Omega \\
& +\int_{\Gamma_{s u}}\left(\frac{\partial \phi_{i}}{\partial x} \phi_{j}+\frac{\partial \phi_{i}}{\partial y} \phi_{j}\right) d s+\int_{\Gamma_{s q}} I\left(\phi_{i}(\mathrm{x})\right) \phi_{j} d s, \\
& \mathcal{F} \mathrm{j}=\int_{\Omega_{s}} f \phi_{j} d \Omega-\int_{\Gamma_{s q}} g \phi_{j} d s, \quad j=1,2, \ldots, n .
\end{aligned}
$$

As for the enforcement of the essential boundary condition, we apply the coupling between the MLPG and finite element method to impose the essence boundary condition, see [8] for detail.

\section{Numerical Test and Discussion}

Several numerical experiments have been performed, in order to illustrate the effectiveness of the MLPG method in electromagnetic scattering by open cavity in the ground plane. For the tests we use the linear basis function and the Gaussian weight function. In all computations, to ensure the invertibility of the moment matrix $A$, we put $r_{i}=2 \times h$, 
where $h_{x}=h_{y}=h$ for simplicity. The calculations are run on a Pentium 4 PC Laptop with $2.50 \mathrm{GHZ}$ of CPU and $4 \mathrm{~GB}$ of RAM.

\subsection{Example 1}

An artificial example defined by Eq. (5) with a cavity $a=b=1$ to verify the accuracy of approximations. The $f(x, y)$ and $g(x)$ are chosen such that the exact function is $u(x, y)=e^{x y} \sin \left(k_{0} x / 2\right) \sin \left(\left(k_{0} / 2+\pi / 4\right) y\right)$, and $g(x)$ is computed by $g(x)=\frac{\partial u}{\partial n}-I(u)$. Accuracy of the estimated solutions can be worked out by measuring the $\left\|e_{n}\right\|_{2}$ and $\left\|e_{n}\right\|_{\infty}$ error norms which are defined by

$$
\begin{gathered}
\left\|e_{n}\right\|_{2}=\left(\int_{\Omega}|u(\mathrm{x})-\hat{u}(\mathrm{x})|^{2} d x d y\right)^{\frac{1}{2}}, \\
\left\|e_{n}\right\|_{\infty}=\max _{\mathrm{x} \in \Omega}\{|u(\mathrm{x})-\hat{u}(\mathrm{x})|\},
\end{gathered}
$$

where $\hat{u}(\mathrm{x})$ is the MLPG solution, and $u(\mathrm{x})$ is the analytic solution. In fact, for obtaining the RCS we pay more attention to the accuracy of the solutions at the aperture $\Gamma$, so the error measures of the numerical solutions on $\Gamma$ are defined by

$$
\begin{aligned}
\left\|e_{\Gamma}\right\|_{2} & =\left(\int_{\Gamma}|u(x, 0)-\hat{u}(x, 0)|^{2} d x\right)^{\frac{1}{2}}, \\
\left\|e_{\Gamma}\right\|_{\infty} & =\max _{(x, 0) \in \Gamma}\{\mid u(x, 0)-\hat{u}(x, 0)\} .
\end{aligned}
$$

The geometry and node distribution $(25 \times 25)$ for the cavity model in the present work are displayed in Fig. 2. We distribute the uniform elements near $\partial \Omega \backslash \Gamma$ so as to apply the coupled technique between the MLPG and finite element method for enforcing the essential boundary conditions. The cavity considered is an empty cavity, i.e. $\varepsilon_{r}=1$. When $k_{0}=2 \pi$ and $4 \pi, 25 \times 25,33 \times 33,49 \times 49,65 \times 65$ nodes are distributed separately. At normal incidence, the results in terms of the errors in the domain and at the aperture are reported in Table 1 and Table 2 respectively, which show the errors become less when more nodes are set, and error performance with different wave number $k_{0}$ goes in a similar manner. The numerical solutions gradually converge to the exact values as the number of nodes increases.

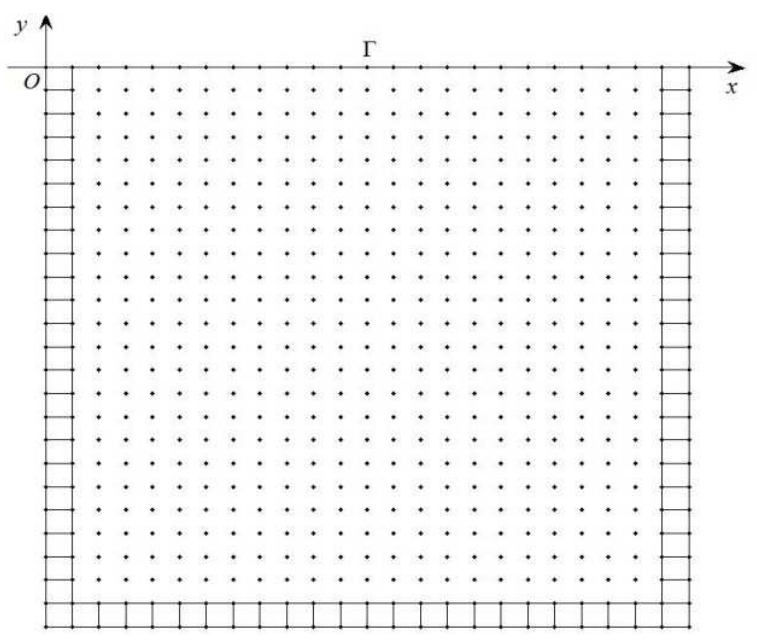

Figure 2. The distribution of nodes and elements for the cavity.

\subsection{Example 2}

A plane wave scattering from a rectangular cavity with 1 meter wide and 0.25 meters deep at normal incidence. We applied the MLPG method to solve the cavity scattering in the TM case. The magnitude of the field of the cavity filled with the medium $\varepsilon_{r}=2.0$ and $\varepsilon_{r}=8+\mathrm{i}$ are given when $k_{0}=2 \pi$ at normal incidence. The radar cross section is also computed, and the results is shown in Fig. 3 and Fig. 4. In these figures, the meshless solutions are compared with those results, noted by ' $\mathrm{o}$ ', solved by the finite element method in [10].

Table 1. Errors of numerical solution for Example 1 when $k_{0}=2 \pi$

\begin{tabular}{lllll}
\hline Nodes & $\left\|e_{n}\right\|_{2}$ & $\left\|e_{n}\right\|_{\infty}$ & $\left\|e_{\Gamma}\right\|_{2}$ & $\left\|e_{\Gamma}\right\|_{\infty}$ \\
\hline $25 \times 25$ & $2.411 \times 10^{-2}$ & $4.032 \times 10^{-2}$ & $1.058 \times 10^{-2}$ & $3.472 \times 10^{-2}$ \\
$33 \times 33$ & $7.452 \times 10^{-3}$ & $9.891 \times 10^{-3}$ & $6.478 \times 10^{-3}$ & $8.774 \times 10^{-3}$ \\
$49 \times 49$ & $1.250 \times 10^{-3}$ & $3.031 \times 10^{-3}$ & $1.002 \times 10^{-3}$ & $2.432 \times 10^{-3}$ \\
$65 \times 65$ & $2.039 \times 10^{-4}$ & $4.013 \times 10^{-4}$ & $1.876 \times 10^{-4}$ & $2.363 \times 10^{-4}$ \\
\hline
\end{tabular}

Table 2. Errors of numerical solution for Example 1 when $k_{0}=4 \pi$.

\begin{tabular}{lllll}
\hline Nodes & $\left\|e_{n}\right\|_{2}$ & $\left\|e_{n}\right\|_{\infty}$ & $\left\|e_{\Gamma}\right\|_{2}$ & $\left\|e_{\Gamma}\right\|_{\infty}$ \\
\hline $25 \times 25$ & $6.961 \times 10^{-2}$ & $7.910 \times 10^{-2}$ & $5.432 \times 10^{-2}$ & $7.884 \times 10^{-2}$ \\
$33 \times 33$ & $3.890 \times 10^{-2}$ & $4.412 \times 10^{-2}$ & $2.903 \times 10^{-2}$ & $4.168 \times 10^{-2}$ \\
$49 \times 49$ & $4.312 \times 10^{-3}$ & $6.273 \times 10^{-3}$ & $1.643 \times 10^{-3}$ & $5.422 \times 10^{-3}$ \\
$65 \times 65$ & $5.143 \times 10^{-4}$ & $7.251 \times 10^{-4}$ & $3.284 \times 10^{-4}$ & $5.293 \times 10^{-4}$ \\
\hline
\end{tabular}



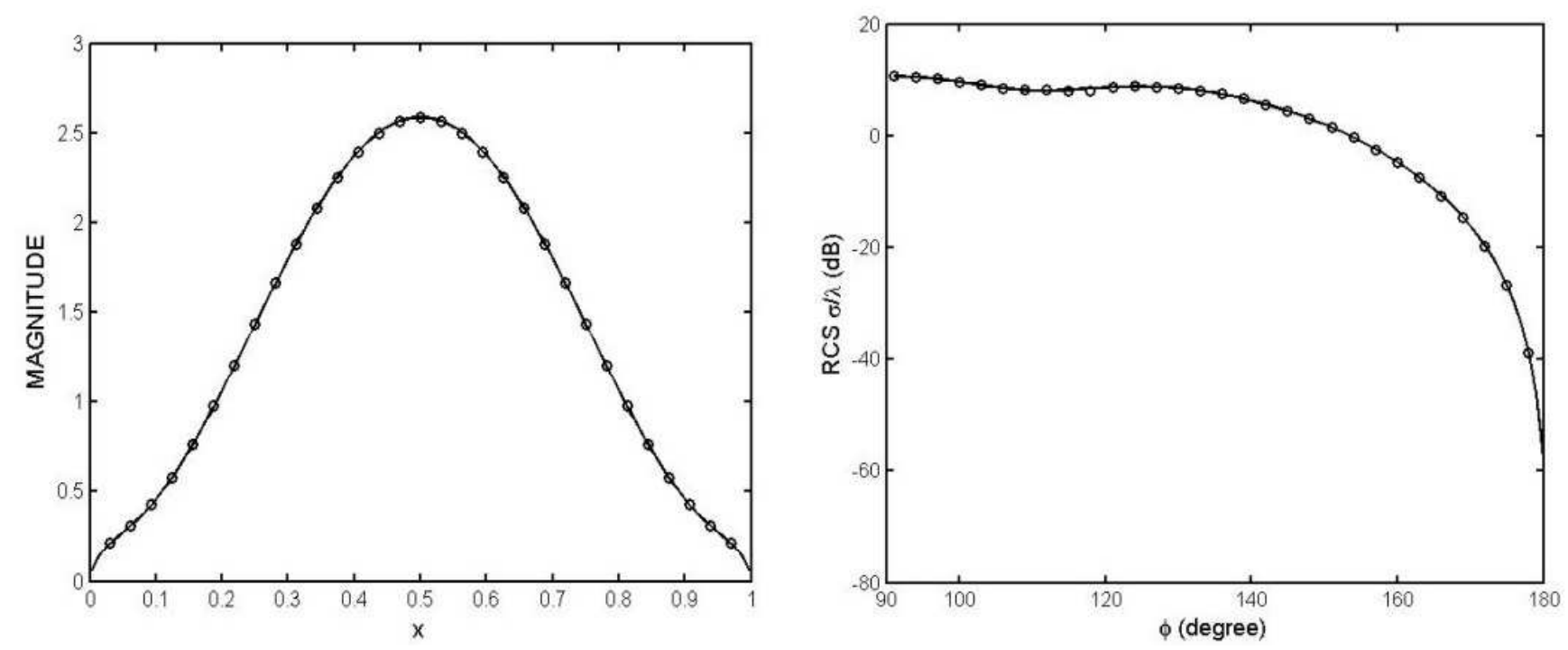

Figure 3. The magnitude of the electric field at the aperture (left) and the RCS (right) for the cavity filled with the medium $\varepsilon_{r}=2.0$.
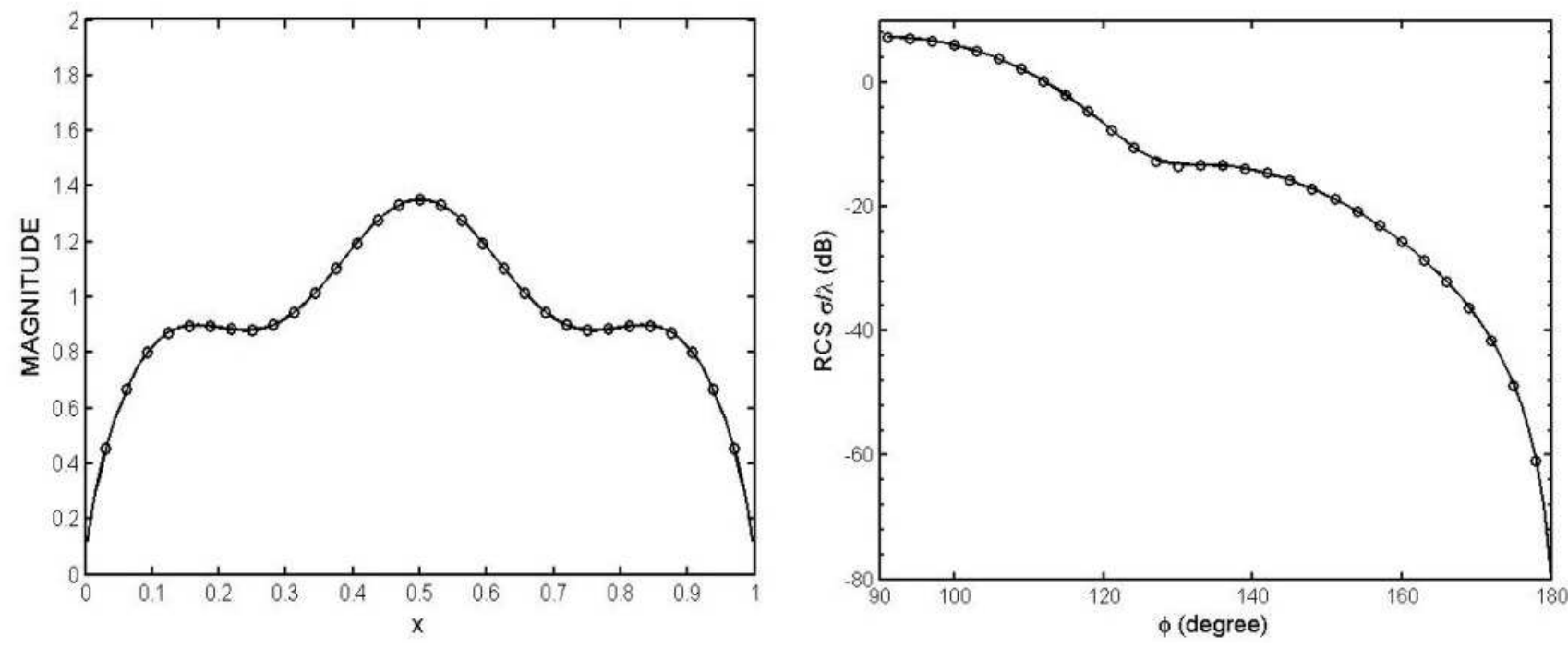

Figure 4. The magnitude of the electric field at the aperture (left) and the RCS (right) for the cavity filled with the medium $\varepsilon_{r}=8+\mathrm{i}$.

\section{Conclusion}

The electromagnetic scattering problems from open cavities have significant application in computational mathematics and electromagnetism. Instead of traditional numerical methods, avoiding reliance on elements or meshes, meshless methods have attracted more and more attention in the engineering and scientific modeling. The meshless local Prtrov-Galerkin method is a truly meshless method in which the trial and test functions are chosen from totally different function spaces. By using local weak form and the moving least square (MLS) approximation, we apply the MLPG method to solve the scattering problem by rectangular cavities in a ground plane in the paper. The results of numerical experiments demonstrate the capability of MLPG method for the scattering problem.

\section{Acknowledgements}

This work was supported by "the Fundamental Research Funds for the Central Universities" (No. 2014ms170).

\section{References}

[1] H. T. Anastassiu, "A review of electromagnetic scattering analysis for intes, cavities, and open ducts," IEEE Antennas Propag. Mag., Vol. 45, pp. 27-40, 2003

[2] H. Ammari, G. Bao and A. W. Wood, "Analysis of the electromagnetic scattering from a cavity," Japan J. Indust. Appl. Math., Vol. 19, pp. 301-310, 2002

[3] J. Liu and J. M. Jin, "A special high-order finite element method for scattering by deep cavity," IEEE Tran. Antennas Propag., Vol. 48, pp. 694-703, 2000 
[4] Z. Xiang and T. Chia, "A hybrid BEM-WTM approach for analysis of the EM scattering from large open-ended cavities," IEEE Trans. Antennas Propag. , Vol. 49, pp. 165-173, 2001

[5] S. N. Atluri and T. Zhu, "A new meshless local Perov-Galerkin approach in computational mechanics," Comput. Mech., Vol. 22, pp. 117-127, 1998

[6] S. N. Atluri and S. Shen, "The meshless local Petrov-Galerkin (MLPG) method: A simple \& less-costly alternative to the finite element and boundary element methods," CMES: Computer Modelling in Engineering, Vol. 3, pp. 11-52, 2002

[7] S. Fernandez-Mendez and A. Huerta, "Imposing essential boundary conditions in mesh-free methods," Computer Methods in Applied Mechanics and Engineering, Vol. 193, pp. $1257-1275,2004$
[8] M. Zhao and Y. Nie, "A study of boundary conditions in the meshless local Petrov-Galerkin (MLPG) method for electromagnetic field computations," CMES: Computer Modelling in Engineering \& Sciences, Vol. 37, pp. 97-112, 2008

[9] J. Wu, Y. Wang, W. Li and W. Sun, "Toeplitz-type aooroximations to the Hadamard integral operators and their applications in electromagnetic cavity problems," Appl. Numer. Math., Vol.58, pp. 101-121, 2008

[10] J. Jin, "The Finite Element Method in Electromagnetics," John Willey Sons, New York, 1993 